\title{
EL SUELO DE LA NATURALEZA COMO LUGAR DE ARRAI GO DE LA CULTURA
}

\author{
Graciela Ralón de Walton \\ Universidad Nacional de San Martín, Argentina \\ grwalton@fibertel.com.ar
}

La obra de Merleau-Ponty ha sido conocida, principalmente, por sus esfuerzos para superar el dualismo antropológico que, en la historia de la filosofía, ha sido sistematizado como cuerpo-alma o espíritu-materia. Sin embargo, la lectura de los escritos posteriores a La estructura del comportamiento y a la Fenomenología de la percepción, en especial, la lectura de los Resúmenes de curso dictados en el Colegio de Francia durante los años 1952-1960, de los cuales en los últimos años han sido publicadas notas que ofrecen una ampliación y precisión del alcance de la temática tratada por el autor, nos proporciona elementos para comprender que la preocupación filosófica de Merleau-Ponty no se restringe solo al plano antropológico, sino que, a través de esas búsquedas, vuelve a plantear uno de los problemas centrales de la filosofía: la relación entre la convicción en la creencia en el mundo, esto es, "la fe perceptiva" y la creación de objetos ideales que constituyen el mundo cultural. Esta problemática responde a la necesidad de mostrar que una de las tareas de la filosofía es comprender que el orden natural y el orden cultural pertenecen al mismo mundo. Lo sensible y lo inteligible no deben ser vistos como órdenes separados: en el paso de lo sensible a lo inteligible "hay doble referencia de mi vida al mundo mudo de la pasividad y al universo voluntariamente retomado por la palabra, la acción y el conocimiento, duplicidad que no es dualismo, puesto que (es) el mismo hombre el que ve y habla $[\ldots]^{\prime \prime 1}$. En un currículum que presenta ante la Sorbona con el nombre de Títulos y trabajos, el autor reconoce que el hom-

\footnotetext{
${ }^{1}$ Maurice Merleau-Ponty, Notes de cours au Collège de France 1958-1959 et 19601961, Paris, Gallimard, 1996, p. 44.
} 
bre que percibe no se limita a ejercer pasivamente los medios naturales que el cuerpo le ofrece para comunicarse con las cosas, sino que "[...] se vuelve sobre las relaciones en las que la percepción lo ha iniciado, se esfuerza por fijarlas, piensa, habla, agrega a los gestos naturales [...] una gesticulación e instituciones lingüísticas, a la naturaleza una cultura"².

Desde un comienzo queremos señalar que, desde el punto de vista fenomenológico, la experiencia perceptiva nos pone en presencia del momento en que para nosotros aparecen "las cosas, las verdades y los bienes, nos da un logos en estado naciente [...]"3, y nos incita hacia el conocimiento y la acción. Con otras palabras, esta experiencia debe ser tratada como primordial no en el sentido de que el conocimiento o la acción se derivan de ella por mera transformación o evolución, sino porque la experiencia de la percepción nos revela los datos permanentes del problema que la cultura intenta resolver. "Hay en los confines de la primera percepción del niño algo así como una presencia todavía desconocida, pero irrefutable, que el conocimiento determinará y llenará a continuación"4. La primacía otorgada a la percepción reside en el hecho de que es una experiencia fuente, lo cual significa que la percepción se presenta como el arquetipo del encuentro originario con el mundo.

Por su parte, el mundo cultural debe ser reconocido como un orden original de advenimiento que no se reduce al orden empírico de los acontecimientos. Sin embargo, lo difícil en este punto es comprender que este universo de sentido o campo de significaciones no constituye un orden autónomo. El universo de la cultura es esencialmente histórico, y la historia no es ni una novedad constante, ni una repetición constante, sino el movimiento único que crea formas estables y las rompe. Así como lo propio de la reflexión acerca de la experiencia perceptiva es haber descubierto la pertenencia de los hombres al mundo y realizar su descripción sin recurrir al pensamiento discursivo, del mismo modo el estudio del pensamiento y de la cultura debe evitar tanto la tentación de una construcción especulativa, que

\footnotetext{
2 Maurice Merleau-Ponty, Parcours deux 1951-1961, Verdier, Lagrasse, 2000, p. 23.

3 Maurice Merleau-Ponty, Le primat de la perception et ses conséquences philosophiques, Grenoble, Cynara, 1989, p. 67.

${ }^{4}$ Maurice Merleau-Ponty, Phénoménologie de la perception, Paris, Gallimard, 1945, p. 378.
} 
superponga "al flujo de la experiencia perceptiva un Pensamiento universal" ${ }^{5}$, como la tentación de reducir los hechos del lenguaje o de la historia a una suma de eventos que resulten de una consideración inductiva o científica.

La fenomenología merleaupontiana ofrece una serie de elementos profundos para pensar el tópico naturaleza-cultura ${ }^{6}$. En este trabajo, nos proponemos considerar que la profundización de la relación entre "cuerpo natural" y "cuerpo cultural", propuesta por Merleau-Ponty, desde La estructura del comportamiento ${ }^{7}$, exige "el redescubrimiento de la Naturaleza paranosotros como suelo de toda nuestra cultura"8.

Tomaremos como punto de partida los análisis acerca del espacio, con la intención de elucidar que sólo es posible ubicar un objeto como próximo o lejano, a nuestra derecha o a nuestra izquierda, en la medida en que nuestro cuerpo anclado en el mundo actúa como punto de orientación; más precisamente, "no habría dirección sin un ser que habitara el mundo y que, por su mirada, trazará en él la primera dirección que será punto de referencia" ${ }^{9}$.

\footnotetext{
${ }^{5}$ Maurice Merleau-Ponty, Parcours deux 1951-1961, p. 24.

${ }^{6}$ Sobre el tema de una fenomenología de la cultura se puede consultar el excelente libro de Javier San Martín Sala Teoría de la cultura, Madrid, Síntesis, 1999. En el capítulo segundo el autor expone desde la perspectiva de Ortega, Heidegger y Husserl el modo en que debe ser abordada la cultura. Según el autor, "la obra de Husserl está atravesada por una columna vertebral: el tópico Natur/Geist, naturaleza, espíritu" (cfr., p. 19). Esta afirmación puede hacerse extensiva, según la perspectiva de este trabajo, a la obra de Merleau-Ponty.

7 En La estructura del comportamiento, Merleau-Ponty advertía acerca de la necesidad de profundizar la distinción entre "cuerpo natural" y "cuerpo cultural". Es necesario tener presente que, a fin de establecer un contraste entre ambos, MerleauPonty se apoya en la diferenciación husserliana entre "pasividad originaria" y "pasividad secundaria". Mientras que el "cuerpo natural" está siempre ahí para la conciencia como algo constituido de antemano, el "cuerpo cultural" se muestra como el resultado de la sedimentación de actos de espontaneidad. Pero la diferencia no es, como en Husserl, una consecuencia de la oposición entre asociaciones que tienen lugar en el ámbito de la síntesis pasiva y asociaciones que dependen de operaciones activas del yo. Más bien se enlaza con diferentes niveles en el desarrollo dialéctico de modo que se relativizan los conceptos de alma y cuerpo.

${ }^{8}$ Maurice Merleau-Ponty, Notes de cours au Collège de France 1958-1959 et 1960-1961, p. 44.

${ }^{9}$ Maurice Merleau-Ponty, Phénoménologie de la perception, p. 491.
} 
Asimismo, las descripciones acerca de las diferentes modalidades antropológicas del espacio, como las de la noche o el sueño, nos permitirán ilustrar que mi cuerpo “habita el espacio y el tiempo"10. En relación con este punto, nos interesa mostrar que de la autoduplicación de una existencia física que ve y toca, y al mismo tiempo es vista y tocada, surge una duplicación espacial que pone de manifiesto, que el "aquí" espacial adquiere rasgos de un no-lugar ${ }^{11}$.

Sin embargo, en la Fenomenología de la percepción, la noción de suelo es considerada como pre-mundo o "mundo natural" cuyo correlato es el cuerpo natural como posibilidad del mundo cultural. En los últimos escritos, Merleau-Ponty emprende la tarea de mostrar que lo que está por debajo no es sólo una vivencia psicológica sino un modo de ser original. En la segunda parte, intentaremos dilucidar que la implantación corporal en la Naturaleza gira alrededor de la interpretación ontológica de la noción de Tierra, a través de la cual, Merleau-Ponty descubre un nuevo tipo de ser ${ }^{12}$. Desde esta perspectiva, el autor señala que

“La toma de conciencia de un Boden, de una sedimentación, podría ser [...] el redescubrimiento de la Naturaleza-para-nosotros como suelo de toda nuestra cultura, y donde echa raíces en particular toda nuestra actividad creadora que no es incondicionada, que debe mantener la cultura en contacto con el ser bruto, y confrontarla con él". ${ }^{13}$

\section{La experiencia perceptiva como suelo de la existencia corporal}

Lo primero a considerar es que el cuerpo expresa de manera eminente el entrecruzamiento entre naturaleza y cultura. Por un lado, el cuerpo se limita a los gestos naturales necesarios para la conservación de la vida y, por

\footnotetext{
${ }^{10}$ Ibid., p. 162.

11 Bernhard Waldenfels, "El habitar físico en el espacio", en Gerhart Schröder / Helga Breuninger (eds.) Teoría de la cultura. Un mapa de la cuestión, Buenos Aires, Fondo de Cultura Económica de Argentina, 2005, pp. 167-169.

12 Según Mauro Carbone, el tipo de ser que Merleau-Ponty reconoce en la descripción husserliana de la Tierra es el ser bruto o salvaje. Como "posibilidad abierta a la corporalidad y a la intersubjetividad" (M. Merleau-Ponty, Parcous deux 1951-1961, p. 232), el mundo percibido pierde el carácter de correlato de los proyectos del sujeto que Merleau-Ponty le había atribuido en la Fenomenología de la percepción (cfr. Mauro Carbone, La visibilité de I'invisible, Olms, Hildestein, 2001, pp. 107s).

${ }^{13}$ Maurice Merleau-Ponty Notes de cours au Collège de France 1958-1959 et 1960-1961, p. 44.
} 
el otro, al producir en ellos una metamorfosis por la que se pasa de un sentido propio a un sentido figurado, abre a nuevas significaciones. MerleauPonty insiste que esta metamorfosis es posible gracias a la doble función del cuerpo. Así, el cuerpo natural o habitual, que incluye la naturaleza con sus estructuras biológicas previas, contiene las disposiciones que son activadas por el cuerpo actual que opera en nuestra actividad. En tanto sostén de estas capacidades, puede ser considerado desde un punto de vista natural como prehistoria y desde un punto de vista cultural como historia. Con otras palabras, se puede distinguir una adquisición pasiva que precede a la propia actividad y remite a la prehistoria de la persona y una adquisición activa que proviene de anteriores actividades y concierne a la historia personal. La apropiación de nuestra corporalidad, que no abandona nuestra situación de hecho, sino que la incluye como un caso particular en el sistema de las otras situaciones posibles, “[...] comienza desde el momento en que nosotros señalamos con el dedo un punto del espacio, pues el gesto de señalar [...] nos supone ya instalados en lo virtual, al final de la línea que prolonga nuestro dedo, en un espacio centrífugo o de cultura"14.

Esta afirmación, que a nuestro entender expresa, implícitamente, la relación entre lo natural y lo cultural, exige tener presente las conclusiones a las que Merleau-Ponty llega en el tratamiento de la espacialidad. En el capítulo dedicado al espacio en la Fenomenología de la percepción, MerleauPonty se propone encontrar una "tercera espacialidad" que haga posible comprender que la experiencia del espacio no puede ser alcanzada "ni por la consideración de los contenidos ni por la consideración de una actividad pura de enlace"15. Las experiencias de Stratton y Wertheimer son reseñadas con la intención de mostrar la relación orgánica del sujeto y del espacio. Describamos, brevemente, la experiencia de Wertheimer. Por medio de un dispositivo se logra que una persona vea a través de un espejo la habitación en la que se encuentra inclinada en un ángulo de 45 grados en relación con la vertical. Al comienzo de la experiencia, el paisaje le resulta extraño, pues, los hombres y las cosas que observa se le aparecen inclinados. Des-

\footnotetext{
14 Maurice Merleau-Ponty, Parcous deux 1951-1961, p. 42.

15 Maurice Merleau-Ponty, Phénoménologie de la perception, pp. 286s.
} 
pués de algunos minutos, bruscamente, el espectáculo cambia: las paredes, el hombre que ve pasar y el pedazo de cartón aparecen en posición vertical. Todo sucede, comenta Merleau-Ponty, como si los objetos que hasta ese momento aparecían oblicuos en relación con el nivel dado "pretendieran por sí mismos darse direcciones privilegiadas, atrajeran hacia sí la vertical, desempeñaran el papel de 'puntos de anclajes' e hicieran oscilar el nivel establecido anteriormente"16. Merleau-Ponty se propone mostrar que, para comprender en qué medida los niveles espaciales pueden transformarse, es necesario partir de "un absoluto en lo relativo", esto es, de un cuerpo que tome posición en el espacio y se solidarice con las apariencias, pero sin confundirse con ellas ${ }^{17}$. La experiencia de un nivel espacial se establece, pues, cuando arraigamos en algún medio. El nivel espacial consiste en un determinado anclaje de mi cuerpo sobre el mundo. Sin embargo, este anclaje no está determinado de una vez para siempre sino que puede cambiar. Así, lo primero que hay que tener en cuenta es que la experiencia de los niveles espaciales nos remite a una espacialidad originaria previamente dada. "EI nivel primordial está en el horizonte de todas nuestras percepciones, pero es un horizonte que por principio no puede nunca ser alcanzado y tematizado en una percepción expresa"18. Podemos adelantar que, si el cuerpo ofrece a las diferentes direcciones como arriba-abajo, derecha-izquierda, delante-detrás, cercanía-lejanía, "un suelo" o "fondo" para su instauración, lo hace como "una facultad percipiente, en tanto que está establecido en determinado dominio y encastrado con el mundo"19. Desde el momento en que comprendemos que es esencial al espacio estar "ya constituido", no es necesario preguntarse, señala Merleau-Ponty, por qué nuestro cuerpo no tiene dominio sobre el mundo en todas las posiciones, o por qué su coexis-

\footnotetext{
${ }^{16}$ Ibid., pp. 287s.

17 Husserl designa al cuerpo propio como punto cero en la medida en que, en tanto constituye el sistema de orientación, no puede ocupar ningún "lugar" en el espacio: "[...] mi cuerpo en su aparición cero (Nullerscheinung) es el miembro central de la aparición del mundo" (Edmund Husserl, Zur Phänomenologie der Intersubjeitivität. Erster Teil, Husserliana XIII, The Haag, Martinus Nijhoff, 1973, p. 280).

18 Ibid., p. 293.

19 Ibid., p. 323.
} 
tencia con el mundo polariza la experiencia y hace surgir una dirección ${ }^{20} ; \mathrm{y}$, a continuación agrega: “la experiencia perceptiva nos enseña, por el contrario, que están presupuestos en nuestro encuentro primordial con el ser y que ser es sinónimo de ser situado"21.

Ahora bien, una vez que se ha puesto en claro que la experiencia de la espacialidad está referida a nuestra fijación en el mundo, es necesario, dice Merleau-Ponty, considerar una espacialidad original para toda modalidad de esta fijación. La experiencia del sueño, de la noche, de la locura o del mito, como modalidades antropológicas del espacio pone de manifiesto que cada una de ellas aparece construida sobre el espacio natural. Más precisamente, es necesario comprender "cómo, por un solo movimiento, la existencia proyecta en torno suyo mundos que me ocultan la objetividad, y le asignan como objetivo a la teleología de la conciencia el destacar estos 'mundos' sobre el fondo de un único mundo natural”22. La relación entre el espacio como modalidad originaria de la percepción y el espacio antropológico nos ofrece, una vez más, la posibilidad de considerar el entrecruzamiento entre el cuerpo natural y el cuerpo cultural. Si bien es cierto que el análisis de la espacialidad pone de manifiesto la facticidad de un "pacto" entre la percepción y el cuerpo como sistema de funciones anónimas, esto es, como cuerpo natural, una nueva orientación reclama un cuerpo virtual, cuyo lugar fenoménico está definido no sólo por su situación sino por su acción. Es necesario no perder de vista que la motricidad constituye una esfera primaria de acceso al mundo. Así, en la experiencia de Wertheimer, la nueva configuración del espacio se produce cuando "el cuerpo virtual desplaza al cuerpo real a tal punto que el sujeto no se siente ya en el mundo en que efectivamente está, y en vez de sus piernas y brazos verdaderos, siente piernas y brazos que sería necesario tener para caminar y actuar en la habitación re-

\footnotetext{
${ }^{20}$ Cfr. Maurice Merleau-Ponty Phénoménologie de la perception, p. 291.

${ }^{21}$ I bid., p. 293. Según Claude Lefort, si bien Merleau-Ponty no ha hablado de un sentido de la orientación, se encuentran numerosas indicaciones que no hace dudar que él "descubrió en el fenómeno una relación originaria con el Ser". Cfr. Claude Lefort, "El sentido de la orientación", en Merleau-Ponty. Notes de cours sur I'origene de la géométrie de Husserl. Suivi de recherches sur la phénoménologie de Merleau-Ponty, Paris, Presses Universitaires de France, 1998, pp. 221-238.

${ }^{22}$ Maurice Merleau-Ponty, Phénoménologie de la perception, p. 340.
} 
flejada"23. Con otras palabras, la historia personal es la continuación de una prehistoria cuyos resultados adquiridos utiliza, poniendo en evidencia que "mi existencia personal es la reasunción de una tradición prepersonal"24. Así, podemos reconocer en los sueños una "significación vital" de lo alto y de lo bajo, que se manifiesta en las poluciones sexuales o en los ritmos respiratorios concomitantes y, "una significación simbólica" que aparece bajo la forma de una imagen, por ejemplo, “la de un pájaro que vuela, y, que, alcanzado por una bala de fusil cae y se reduce a un montículo de papel negro"25. Tanto el movimiento hacia lo alto en el espacio físico como el deseo "son simbólicos uno del otro". En ambos casos se expresa "la estructura esencial de nuestro ser como ser situado en relación con un medio"26. B. Waldenfels analiza el entrecruzamiento entre la significación vital y la significación simbólica apoyándose en investigaciones del paleontólogo André Leroi-Gourhan. Esto le permite referirse a una "estética" en un sentido muy amplio con base en una "estética fisiológica" ligada a ritmos y repeticiones que se encuentran en una zona de cruce de naturaleza y cultura, es decir, una naturaleza carente de historia y una cultura arcaica que toma la naturaleza como medida ${ }^{27}$. Del mismo modo que en el aprendizaje del lenguaje se reconocen estructuras sonoras o imágenes escritas anteriores a toda comprensión de un significado pero que, sin embargo, constituyen el paso previo a la comprensión del significado, es posible también encontrar en el ritmo del movimiento formaciones de sentido. No se podría intencionar o comprender algo como algo si no se repitiera en el ir y venir del movimiento. Se trata de ritmos que articulan el movimiento y que no tienen un significado ni tienden a una meta como lo hace una fase en el desarrollo del organismo, sino que reciben una determinación a partir del modo en que transcurre el movimiento. Lo que importa no es la meta sino el cómo de la movilidad. Para que haya ritmo es imprescindible una duración, una repeti-

\footnotetext{
23 Ibid., p. 289.

24 Ibid., p. 293

${ }^{25}$ Maurice Merleau-Ponty, Phénoménologie de la perception, p. 329.

26 Idem.

27 Cfr. Bernhard Waldenfels, Sinnesschwellen. Studien zur Phänomenologie des Fremden 3, Frankfurt am Main, Suhrkamp, 1999, pp. 53-85.
} 
ción y un ordenamiento o relación numérica según la cual se orienta la repetición. El ritmo tiene que ver con unidades de tiempo y movimiento que son numerables y su reiteración implica un retorno de lo desigual como igual. Como articulación del movimiento, el ritmo se encuentra en las esferas de los diversos sentidos e implica una forma, es decir, un modo de fluir en medio de un elemento móvil. En virtud de la articulación rítmica surgen movimientos determinados que se suceden en el flujo indiferenciado del acontecer. Estas formas tienen el carácter de símbolos rítmicos en que un movimiento remite a los otros movimientos que integran el acontecer rítmico. Los diferentes pasos del movimiento no se suceden como una suma en que un elemento de movimiento se añade a otro sino que configuran una estructura en que los momentos se encuentran en tensión. En el plano corporal aparecen los ritmos de la inspiración y la expiración, de las pulsaciones y los latidos del corazón, del sueño y la vigilia, de la alimentación y el hambre. Estos ritmos se enlazan con los ritmos cósmicos del día y la noche, de las mareas, de los cambios del tiempo y de las estaciones. Por otra parte, los ritmos normales o cotidianos pueden ser perturbados por padecimientos y defectos físicos y, además, pueden ser interrumpidos a propósito por prácticas extraordinarias que recurren al ayuno y al insomnio provocado. Merleau-Ponty había anticipado esta clase de consideraciones al advertir que los estudios realizados por la psicología animal y la etnología descubren en la animalidad esbozos, prefiguraciones parciales y como caricaturas anticipadas: “El hombre y la sociedad no están exactamente fuera de la naturaleza y de lo biológico: se distinguen más bien reuniendo las 'apuestas' de la naturaleza y arriesgándolas todas en conjunto"28.

Es necesario insistir, pues, en que lo biológico y lo cultural no deben ser considerados como capas superpuestas sino que la existencia corporal oscila entre una y otra. En virtud de la autodiferenciación del cuerpo, la distinción entre los planos de lo natural y lo cultural resulta superflua y abstracta. Hay una condición ambigua del cuerpo que no se puede subordinar unívocamente ni a la naturaleza ni a la cultura. Es importante tener en cuenta que el cuerpo propio interviene en la autodiferenciación entre cuerpo propio (Leib)

${ }^{28}$ Maurice Merleau-Ponty, Signes, Paris, Gallimard, 1960, p. 157. 
y cuerpo físico (Körper). No se trata de dos polos de una diferencia que pueden intercambiarse, sino que el cuerpo propio tiene una primacía sobre el cuerpo físico en el sentido de que opera en la autodiferenciación. El cuerpo propio, que es vidente y visible, mediante una naturalización se convierte en una cosa física que es visible, pero no vidente. Se produce una abstracción por la cual el cuerpo propio se encuentra entre los otros cuerpos físicos de la naturaleza y los momentos naturales de mi corporalidad se comparan entonces con los procesos de los cuerpos inertes. Por otro lado, mediante una espiritualización, el cuerpo propio se convierte en un yo espiritual que es vidente, pero no visible. A la abstracción física se añade una segunda abstracción por la cual surge un yo puramente espiritual carente de cuerpo. Permanecer en una de esas abstracciones, es decir, en lo físico o en lo espiritual, sin advertir lo que se encuentra en la raíz de ellas, es convertir la autodiferenciación en cuerpo propio y cuerpo físico en una autoescisión en que los términos de la diferencia son aprehendidos en manera absoluta ${ }^{29}$.

Ejemplificaremos a través de la vivencia del espacio cómo en virtud de la autodiferenciación del cuerpo propio podemos oscilar entre el espacio físico en el cual estamos y el espacio cultural o virtual. Merleau-Ponty describe esta posibilidad en los siguientes términos: “Llego a un pueblo para pasar las vacaciones, me instalo en él y se convierte en el centro de mi vida. La falta de agua pasa a ser un acontecimiento. Sin embargo, si escucho por la radio que en la ciudad donde habito hay amenazas de guerra, repentinamente, comienzo a sentirme en este lugar como un exiliado o un excluido"30. Con otras palabras, si bien nuestra percepción y nuestro cuerpo nos habitúan a considerar como centro del mundo la configuración que ellos nos ofrecen aunque no sea, efectivamente, la nuestra, es posible “ 'estar en otra parte' aunque esté aquí, y si se me mantiene lejos de los que amo me

29 Cfr. Bernhard Waldenfels, Das leibliche Selbst. Vorlesungen zur Phänomenologie des Leibes, Frankfurt am Main, Suhrkamp, 2000, pp. 252-257.

30 Maurice Merleau-Ponty, Phénoménologie de la perception, p. 330. Es necesario tener en cuenta que el movimiento pone en evidencia la implicación del espacio y del tiempo. "Las cosas coexisten en el espacio porque están presentes al mismo sujeto que percibe y envueltas en una misma onda temporal" (Ibid., p. 318). 
siento excéntrico con la verdadera vida" ${ }^{31}$. Esta doble manera de habitar el espacio, que se corresponde con la autodiferenciación del cuerpo propio, hace comprensible que, en los primeros inicios, el género humano haya oscilado entre el nomadismo y el sedentarismo. "Sentido de posibilidad" y "sentido de realidad", comenta B. Waldenfels, no se contradicen, pero sí pueden excluirse uno a otro. "Si aumenta demasiado el 'sentido de realidad', entonces nos acercamos a una realidad sin posibilidades abiertas, si, por el contrario, aumenta demasiado el 'sentido de posibilidad', corremos el riesgo de una virtualidad sin anclaje en la realidad"32.

\section{La Tierra como suelo de experiencia: suelo natural y suelo cul tural}

En las notas que amplían los cursos sobre la naturaleza Merleau-Ponty afirma:

“Es naturaleza lo primordial, es decir, lo no construido, lo no-instituido; de ahí la idea de una eternidad de la naturaleza (eterno retorno), de una solidez. La naturaleza es [...] nuestro suelo, no lo que está delante sino lo que nos lleva (ce qui nous porte)". ${ }^{33}$

En tanto suelo la naturaleza exhibe las características que Husserl le asigna a la noción de Tierra. Así, el interés principal que guía el análisis retrospectivo llevado a cabo en "El filósofo y su sombra" es poner de manifiesto la presencia de seres que "nutren secretamente" nuestras idealizaciones y objetivaciones. Entre ellos, se encuentra la Tierra, que “[...] no está en movimiento como los cuerpos objetivos, pero tampoco en reposo, puesto que no se ve a qué estaría 'fijada'. Ella es así 'suelo' o ‘cuna' de nuestro pensamiento como de nuestra vida [...]"34. Asimismo, en el comentario a El origen de la geometría, Merleau-Ponty interpreta que la nociones de apertura y horizonte empleadas en el nivel de la idealidad pueden ser vueltas a encontrar "desde abajo", lo cual es ilustrado, nuevamente, con las descripciones realizadas por Husserl en el manuscrito "Giro de la teoría copernica-

\footnotetext{
31 Ibid., p. 330.

32 Bernhard Waldenfels, “El habitar físico en el espacio", p. 170.

${ }^{33}$ Maurice Merleau-Ponty, La nature. Notes. Cours du Collège de France, Paris, Seuil, 1995, p. 20.

${ }^{34}$ Maurice Merleau-Ponty, Signes, p. 227.
} 
na". Estas investigaciones ponen al descubierto, un modo de ser del que nos hemos distanciado: "el ser del 'suelo' (Boden), y, en primer lugar, el de la Tierra $[\ldots]^{33}$.

Resulta interesante destacar que el escrito de Husserl ya había sido leído por Merleau-Ponty en la época en que escribe la Fenomenología de la percepción. El siguiente texto da cuenta de ello:

“no habría movimiento efectivo y no tendría la noción de movimiento si, en la percepción, no se pusiera la tierra como 'suelo' de todos los reposos y de todos los movimientos, más acá del movimiento y del reposo, porque la habito; e igualmente no habría dirección sin un ser que habitara el mundo y que, por su mirada, trazara en él la primera dirección que será punto de referencia". ${ }^{36}$

Sin embargo, la noción de suelo en esa obra es atribuida, como hemos comentado en la primera parte, a "un espíritu cautivo" o "natural" como "sistema de funciones anónimas que envuelve toda fijación particular en un proyecto general"37. En los últimos escritos, Merleau-Ponty se interesa por develar las implicancias ontológicas de la noción de Tierra. El descubrimiento de la Tierra como suelo pone en marcha una filosofía del mundo como Offenheit del Umwelt, por oposición al infinito "representado" de las ciencias clásicas de la Naturaleza ${ }^{38}$. La homogenización de lo exterior transformado en objeto, de los astros como objetos y de la tierra como "simple hecho", "todo esto está construido sobre la implantación corporal en un "Ser-Patriaterritorio"39. La idealización de la naturaleza terrestre es una idealización de la relación terrestre con el suelo y de la coexistencia terrestre. Según Merleau-Ponty, Husserl se arriesga a describir la Tierra como "patria e historicidad de los sujetos carnales, como [...] suelo de verdad, o arca que trasporta hacia el porvenir las simientes del saber y la cultura" ${ }^{40}$.

\footnotetext{
35 Maurice Merleau-Ponty, Résumés de tours. Collège de France 1952-1960, Paris, Gallimard, 1968, pp. 168s.

${ }^{36}$ Maurice Merleau-Ponty, Phénoménologie de la perception, p. 491.

37 I bid., p. 280.

38 Maurice Merleau-Ponty, Résumés de cours. Collège de France 1952-1960, p. 170.

39 Maurice Merleau-Ponty, Merleau-Ponty. Notes de cours sur l'origine de la géométrie de Husserl. Suivi de recherches sur la phénoménologie de Merleau-Ponty, p. 170.

40 Maurice Merleau-Ponty, Résumés de cours. Collège de France 1952-1960, p. 116.
} 
Al igual que Husserl, Merleau-Ponty destaca que para el hombre copernicano la tierra es un cuerpo físico (Körper) esférico en el espacio infinito, mientras que para nosotros no es cuerpo físico, sino suelo para la experiencia (Erfahrungsboden). Para Husserl carece de sentido hablar de un espacio universal vacío, como mundo astronómico que ya es infinito o de un espacio que la circunda y donde la tierra estaría como un cuerpo físico más. La tierra no puede ser experimentada como un cuerpo físico porque no puede ser percibida por todos sus lados. Tiene el carácter de un cuerpo-suelo o cuerpo-total. "La tierra es un todo, cuyas partes - cuando ellas son pensadas por sí [...] - son cuerpos físicos, pero ella como 'todo' no es ningún cuerpo físico" ${ }^{41}$.

La tierra es, además, de soporte para todos los cuerpos físicos, un suelo para nuestra corporalidad. Entre el ser de la tierra y mi cuerpo hay un parentesco. Sin embargo, el retroceso al suelo de la tierra nos obliga a considerar en qué sentido el cuerpo puede ser considerado como suelo. Más precisamente, si en la génesis de la experiencia la Tierra aparece como "suelo de la experiencia para todo cuerpo", ¿pierde nuestro cuerpo la dimensión de suelo? Comencemos por tener en cuenta que, al igual que la tierra, mi cuerpo propio posee los mismos rasgos que caracterizan la tierra: no perspectividad (como un todo), centramiento (como punto cero) y firmeza (en tanto inmóvil) ${ }^{42}$. La tierra es un suelo para nuestra corporalidad, esto es, el cuerpo-suelo sobre el cual tiene lugar todo nuestro andar, toda nuestra vida perceptiva natural y originaria. En la medida en que el cuerpo propio está anclado en la tierra, su condición de centro de toda orientación y movimiento encuentra un punto de referencia ulterior. Además, todo movimiento tiene lugar sobre ella o en dirección hacia ella: “[...] la tierra misma no se mueve y no reposa, en relación con ella tienen sentido el reposo y el movi-

\footnotetext{
${ }^{41}$ Edmund Husserl, “Grundlegende Untersuchungen zum phänomenologischen Ursprung der Räumlichkeit der Natur", en Marvin Farber (ed.) Philosophical Essays in Memory of Edmund Husserl, New York, Greenwood Press, 1968, p. 313. Este texto corresponde al Ms. D 17. Traducción castellana de Agustín Serrano de Haro: La tierra no se mueve, Madrid, Universidad Complutense, 1995.

${ }^{42}$ Cfr. Luis Rabanaque, "El papel de la cenestesia en la constitución de la tierra como suelo de experiencia, en Escritos de Filosofía 39-40 (2001) p. 160.
} 
miento"43. No obstante, esta referencia absoluta a la tierra, se debe admitir una cierta relatividad del reposo y el movimiento ya que pueden darse como puntos de referencia para ellos cuerpos-suelos que son experimentados a su vez como cuerpos en reposo o cuerpos móviles en relación con el suelo-tierra que no es experimentado como un cuerpo. Un ejemplo de cuerposuelo relativo se encuentra en un coche en marcha en relación con el cual se puede tener la experiencia de un acercamiento o un alejamiento de otro cuerpo físico ${ }^{44}$. Pero este cuerpo-suelo relativo está siempre referido a la tierra como suelo absoluto.

De esta manera, la tierra se constituye como un sustrato para toda la actividad corporal y como un centro de orientación que está fuera del cuerpo propio.

“Tenemos un espacio alrededor en tanto que sistema de los lugares -es decir como sistema de los fines posibles de los movimientos del cuerpo. Pero en ese sistema, todos los cuerpos terrestres tienen también un 'lugar' particular, salvo la tierra". ${ }^{45}$

En la intuición del espacio (Raumanschauung), del tiempo (Zeitanschauung) de la causalidad natural (Naturkausalität) y aun en el concepto de historia, lo que se revela, insiste Merleau-Ponty, es "un tipo de ser nuevo". Más precisamente, la concepción copernicana pone de manifiesto que se trata de una idealización de la relación terrestre con el suelo y con la coexistencia terrestre: “Que la historia tenga un sentido, que ella sea una, que sea dimensión, esto: esta racionalidad, se forma sobre la relación preobjetiva de los hombres con los hombres y la tierra"46.

Ahora bien, como ya adelantamos, Merleau-Ponty se interesa por hacer explícita la significación filosófica de este tipo de ser puesto de manifiesto por la experiencia del suelo y del cuerpo: “[...] la filosofía como estudio del suelo de sentido (Sinnesboden) es literalmente: Boden natural (la tierra) y

\footnotetext{
${ }^{43}$ Edmund Husserl, “Grundlegende Untersuchungen zum phänomenologischen Ursprung der Räumlichkeit der Natur", p. 309.

44 Ibid., pp. 311 s.

${ }^{45}$ Cfr. Ibid., p. 313.

46 Maurice Merleau-Ponty, Merleau-Ponty. Notes de cours sur l'origine de la géométrie de Husserl. Suivi de recherches sur la phénoménologie de Merleau-Ponty, p. 89.
} 
Boden cultural-histórico construido sobre él"47. Así, las últimas búsquedas se centralizan en "reencontrar este espíritu bruto y salvaje bajo todo el material cultural que se ha dado [...]"48; esto significa el redescubrimiento de una Naturaleza para nosotros como suelo de toda nuestra cultura. El retorno del mundo objetivo al mundo de la vida no sólo alcanza a las cosas percibidas, sino también a las formaciones históricas que han servido para configurar las relaciones entre los hombres.

En el curso dictado en 1958-1959 titulado "La filosofía hoy", MerleauPonty caracteriza nuestro estado de no-filosofía a través de dos rasgos: crisis de la racionalidad en las relaciones entre los hombres y crisis de la racionalidad en nuestras relaciones con la naturaleza. Los avances científicos y técnicos nos sitúan frente a energías que ya no están dentro del marco del mundo y, más aún, podrían destruirlo. A su vez, del hecho de que es el hombre quien elabora la técnica, "un nuevo prometeísmo" se asocia a lo anterior dándole al mundo un "rostro prehumano"49. En razón de esto, Merleau-Ponty afirma, que "un naturalismo extremo y un extremo artificialismo se hallan inextricablemente asociados [...]"50. Así, el pensamiento contemporáneo queda prisionero de la siguiente contradicción: el hombre sólo se atiene a los constructa que el ha producido pero ellos son productos de una naturaleza comprendida según el modelo de las ciencias de la naturaleza. Merleau-Ponty evalúa, que el peligro de esta contradicción se encuentra presente desde el comienzo de la ciencia moderna como:

“1) desvelamiento de una naturaleza en sí, 2) desvelamiento, sin embargo, condicionado por la historia humana. De ahí la idiotez de querer comprender el acontecimiento de la ciencia actual, como hecho de Naturaleza (en sí) en el sentido que la ciencia da a este vocablo". ${ }^{51}$

Esta experiencia pone al descubierto que lo que durante siglos fue considerado por la humanidad como un suelo sólido hoy se revela frágil. Sin embargo, "en el orden de la cultura y de la investigación, la relativización

\footnotetext{
47 Ibid., p. 82.

48 Ibid., p. 274.

${ }^{49}$ Maurice Merleau-Ponty, Résumés de cours. Collège de France 1952-1960, p. 146.

50 Idem.

${ }^{51}$ Maurice Merleau-Ponty, Notes de cours au Collège de France 1958-1959 et 1960-1961, p.
} 43. 
de lo que se creía el suelo de la historia y de la Naturaleza es ya descubrimiento de una nueva solidez" ${ }^{\prime 2}$.

La posibilidad de redescubrir un suelo sólido está relacionada con la posibilidad y el alcance de la filosofía. Así, por no-filosofía, Merleau-Ponty no entiende su anulación o desaparición sino una dimensión que se encuentra por debajo del campo instituido de la filosofía que es, precisamente, el que está en crisis. Es necesario reconocer otros modos de pensamiento que lejos de eliminar la exigencia filosófica, la estimulan a buscar otras vías de reflexión: “La filosofía encontrará ayuda en poesía, arte, en una relación mucho más estrecha con ellas, renacerá e reinterpretará así su propio pasado de metafísica -que no es pasado" 53 .

\footnotetext{
52 Maurice Merleau-Ponty, Résumés de cours. Collège de France 1952-1960, p. 146.
}

53 Maurice Merleau-Ponty, Notes de cours 1958-1961, p. 39. 